\title{
Waist circumference cutoff points for predicting metabolic abnormalities in Lebanese adults
}

Rouham Yamout ${ }^{1}$, Walid Ammar ${ }^{2}$, Jiana Tabbara ${ }^{1}$, Souha Fares ${ }^{1}$, Moubadda Assi ${ }^{1}$, Abla M. Sibai ${ }^{1}$

\author{
${ }^{1}$ Department of Epidemiology and Population Health, American University of Beirut, \\ Beirut, Lebanon \\ ${ }^{2}$ Ministry of Public Health, Beirut, Lebanon
}

Submitted: 22 July 2018

Accepted: 15 April 2019

Arch Med Sci Civil Dis 2019; 4: e64-e71

DOI: https://doi.org/10.5114/amscd.2019.86814

Copyright $\odot 2019$ Termedia \& Banach

\section{Abstract}

Introduction: Central obesity, as measured by waist circumference, performs differently across diverse localities, and there is a need to optimize gender-based cutoff points to specific ethnic and population groups.

Material and methods: A total of 1,528 asymptomatic individuals free from cardiac disease aged 40 years and above and attending 25 primary health care centers distributed over the entire Lebanese territory were recruited for a cardiovascular risk screening service implemented by the Ministry of Public Health in 2012. Using receiver operating characteristics curve analyses, we evaluated different waist circumference cutoff points for the optimal combination of sensitivity and specificity that distinguish men and women with concomitant presence of impaired blood sugar and hypertension.

Results: The optimal waist circumference cutoffs for prediction of the outcome were $98.5 \mathrm{~cm}$ in men and $91.5 \mathrm{~cm}$ in women, yielding better predictive characteristics than those recommended by the International Diabetes Federation (IDF). Based on the study values, the prevalence rates of central obesity in our sample $(36.2 \%$ in males and $40.2 \%$ in females) were lower than those estimated using the IDF cutoff values $(55.2 \%$ and $79.7 \%$, respectively).

Conclusions: Findings from this first examination of optimal central obesity cutoff points in Lebanon confirm the need for nation-wide studies with more inclusive cardio-metabolic outcomes for the development of appropriate screening protocols.

Key words: central obesity, waist circumference, Lebanon, cutoff points.

\section{Introduction}

Cardiovascular diseases (CVD) are the leading cause of morbidity and mortality worldwide and are a major barrier to sustainable human development [1]. Countries with the greatest number of CVD deaths, after accounting for population size, are found throughout the developing world including the Eastern Mediterranean region [2]. Early detection and timely management of CVD risk factors is the cornerstone in the prevention of CVD and for reducing associated health care costs. Among these risk factors, central or abdominal obesity, as measured by waist circumference, is gaining popularity as a key risk factor for CVD. Waist circumference (WC) is a consistent component of all metabolic syndrome definitions $[3,4]$ and a significant predictor of obesity-related metabolic factors [5-7].

\author{
Corresponding author: \\ Prof. Abla M. Sibai \\ Department of \\ Epidemiology and \\ Population Health \\ American University \\ of Beirut \\ 11-0236 Beirut, Lebanon \\ E-mail: am00@aub.edu.lb
}


It is also emerging as a more powerful determinant than body mass index for cardiovascular prognosis in studies conducted worldwide, including developed and developing countries [8].

Measuring WC is a simple procedure limited to the measurement of waistline using an elementary tailor tape and requires minimal training. Also, it entails minimal cost, does not require medical qualification or any kind of calculation or technological infrastructure, and can therefore be used for cardiovascular risk assessment in routine clinical practice or mass screening at any health encounter and at the population level $[9,10]$.

As with other anthropometric indices, WC measurement performs differently across diverse localities and population groups [11, 12] and cannot be useful as a predictor of cardiovascular risk if not optimized to specific ethnic groups and geographical areas [5]. The International Diabetes Federation (IDF) and the World Health Organization (WHO) recommend the use of ethnic specific cutoffs for WC measurement in metabolic syndrome to define a threshold for central obesity [4, 13]. However, in the absence of enough evidence for the Arab population, the IDF and WHO recommend the use of the European definition of central obesity set at WC $\geq 94 \mathrm{~cm}$ for men and WC $\geq 80 \mathrm{~cm}$ for women $[3,13]$.

Consequently, researchers from the Arab region have been increasingly engaged in studies that aim at defining optimal WC cutoff points appropriate to their populations. In these studies, the cutoff points have varied among men between $80 \mathrm{~cm}$ in Oman [14] and $100.5 \mathrm{~cm}$ in Egypt [15], and among women between $84.5 \mathrm{~cm}$ in Oman [14] and 96.2 $\mathrm{cm}$ in Iran and Egypt $[15,16]$. While these studies were not always comparable because of the use of different outcome measures against which the WC cutoff points were optimized and owing to the various age groups targeted in the study population, the findings elicited a set of concerns. These revolved around the applicability of the European WC cutoffs to the Arab population considering two arguments: firstly, almost none of the studies obtained WC cutoffs that were comparable to the recommended European ones, and secondly, the cutoff points obtained were varied, suggesting that the region is non-homogeneous with regard to average population values of WC.

Using data from a large database drawn from a cardiovascular risk screening intervention conducted in primary care centers in Lebanon, this study was set with the goal of optimizing the use of WC measurements in screening protocols in the country. It aimed at identifying the optimal WC cutoff points that best discriminate otherwise healthy men and women presenting concomitantly with impaired blood sugar and elevated blood pressure. Findings from this study are of significance to warrant the assessment of optimal WC cutoff points in nationally representative community-based populations for assessment of cardiovascular risk.

\section{Material and methods}

\section{Study subjects}

This study is based on secondary analysis of data generated as part of a screening service implemented by the Lebanese Ministry of Public Health in 2012 in 25 primary health care centers distributed over the entire Lebanese territory. The detailed description of the study design, conduct and the preliminary results are reported elsewhere [17]. In brief, the screening served 5,875 beneficiaries and included questions and direct measurements to assess six significant cardiovascular risk factors (random blood sugar (RBS), blood pressure (BP), smoking status, family history of premature CVD, body mass index (BMI), and WC), along with age, sex, education, work status and residence. Fieldwork and assessments were undertaken by 72 trained non-physician health workers working at the respective centers.

The beneficiaries of the program were recruited purposefully with close to two-thirds of them being recruited while attending the health center for services not related to chronic conditions ( $n=$ $3,555 ; 60.5 \%)$. The remaining subjects underwent the screening protocol in their residence through home visits performed by the health workers. For the parameters included in this study, the protocol was thoroughly identical in both settings. For the objectives of this study, a total of 1,528 records were retained for the analysis. These belonged to individuals fasting for $>8 \mathrm{~h}$, aged 40 years and above, with no prior history of diabetes, hypertension, dyslipidemia or documented CVD, and with no missing data for variables pertinent to the study (fasting blood sugar (FBS) testing, BP, BMI and WC measurements reports).

\section{Variables and measurements}

The outcome variable was defined as the concomitant presence of two metabolic abnormalities: impaired sugar metabolism defined as FBS $>110 \mathrm{mg} / \mathrm{dl}$ and raised blood pressure defined as $\mathrm{BP}>135 / 85 \mathrm{~mm} \mathrm{Hg}$. Fasting participants were screened for RBS through a standardized finger glucometer, while blood pressure was measured in the right arm, using a standardized digital sphygmomanometer. WC was measured at the point midway between the lower rib and the iliac crest, using a regular tape. Central obesity was then defined according to the IDF categorization (WC $\geq 94 \mathrm{~cm}$ in men and $\geq 80 \mathrm{~cm}$ in women), and 
according to the optimal WC cutoffs as obtained from subsequent analysis of the receiver operator characteristic (ROC) curve.

\section{Ethical approval}

De-identified clinical data were retrieved from the health information system of the Primary Health Care (PHC) department at the Ministry of Public Health.

\section{Statistical analysis}

Continuous variables were reported as means and standard deviations (SD), and comparisons across groups were carried out using the independent samples $t$-test. Categorical variables were summarized using frequencies and proportions and were compared using the $\chi^{2}$ test. All data were presented for men and women separately. The ROC curve was plotted between the test variable (WC) and the state variable (metabolic outcome) to determine the optimal WC cutoff, which is the point on the ROC curve closest to the upper left corner with optimal sensitivity and specificity. The overall performance of the different measures of central obesity against metabolic outcome was assessed using the $\kappa$ statistic, and calculation of sensitivity, specificity and Youden's index were retrieved from simple two-by-two tables between the dichotomized central obesity variables. The software IBM SPSS Statistics v. 20 was used for all analyses, and the level of significance was set at $\alpha<0.05$.

\section{Results}

\section{Sample characteristics}

The sample consisted of 536 (35\%) men and 992 (65\%) women with a similar mean age of around 51 years for both sexes (Table I). The mean WC was $95.1 \mathrm{~cm}$ in men and $90.3 \mathrm{~cm}$ in women. While mean FBS levels and proportion of impaired blood sugar were comparable in men and women ( $p=0.861$ and $p=0.850$, respectively), mean systolic (125 mm Hg vs. $121 \mathrm{~mm} \mathrm{Hg}$, respectively; $p<0.001$ ) and diastolic (78 mm Hg vs. $76 \mathrm{~mm} \mathrm{Hg}$, respectively; $p<0.01)$ blood pressure as well as proportion of hypertension ( $28.9 \%$ vs. $22.5 \% ; p=$ 0.012 ) were significantly higher in men compared to women. Overall, $4.3 \%$ had metabolic outcome,

Table I. Baseline study sample characteristics

\begin{tabular}{|lccccccc|}
\hline & \multicolumn{2}{c}{$\begin{array}{c}\text { Men } \\
\text { Parameter }\end{array}$} & \multicolumn{2}{c}{$\begin{array}{c}\text { Women } \\
(n=536)\end{array}$} & \multicolumn{2}{c}{$\begin{array}{c}\text { Total } \\
(n=152)\end{array}$} & \multirow{2}{*}{$P$-value* } \\
\cline { 2 - 7 } & Mean & SD & Mean & SD & Mean & SD & \\
\hline Age [years] & 51.5 & 9.5 & 51.2 & 9.7 & 51.3 & 9.6 & 0.472 \\
\hline WC [cm] & 95.1 & 11.5 & 90.3 & 11.7 & - & - & - \\
\hline FBS [mg/dl] & 99.2 & 19.0 & 99.0 & 19.8 & 99.0 & 19.5 & 0.861 \\
\hline SBP [mm Hg] & 125.1 & 14.6 & 121.4 & 14.6 & 122.7 & 14.7 & $<0.001$ \\
\hline DBP [mm Hg] & 78.0 & 9.5 & 75.8 & 9.9 & 76.5 & 9.8 & $<0.001$ \\
\hline Parameter & $n$ & $\%$ & $n$ & $\%$ & $N$ & $\%$ & $P$-value* \\
\hline Impaired blood sugar (FBS $>110 \mathrm{mg} / \mathrm{dl})$ & 54 & 10.1 & 103 & 10.4 & 157 & 10.3 & 0.850 \\
\hline Hypertension (BP $>$ 135/85 mm Hg) & 155 & 28.9 & 223 & 22.5 & 378 & 24.7 & 0.012 \\
\hline Metabolic outcome (elevated FBS and BP) & 24 & 4.5 & 41 & 4.1 & 65 & 4.3 & 0.753 \\
\hline
\end{tabular}

WC - waist circumference, FBS - fasting blood sugar, SBP - systolic blood pressure, DBP - diastolic blood pressure, BP - blood pressure. ${ }^{*} P$-values obtained using $t$-test and $\chi^{2}$ test for continuous and categorical variables, respectively.
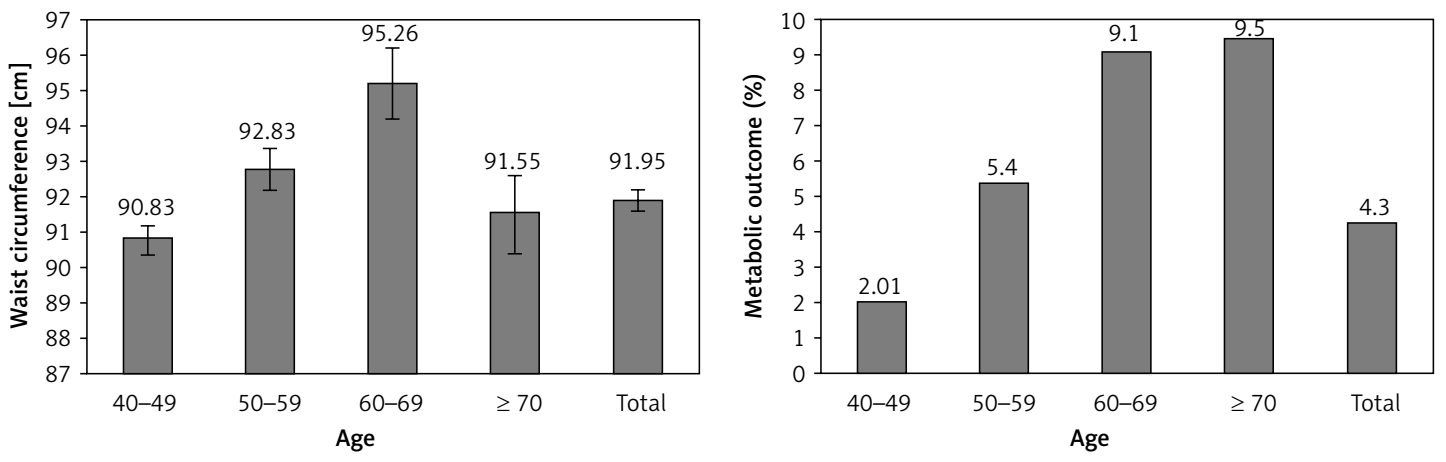

Figure 1. Mean waist circumference and metabolic outcome by age 
defined here as elevated FBS and BP. Whereas metabolic outcome increased consistently with age, mean WC value was highest in the age group 60-69 years and decreased thereafter (Figure 1).

\section{ROC curve analysis and predictive characteristics}

The ROC curve was plotted to determine the discriminative values of WC that best predicts

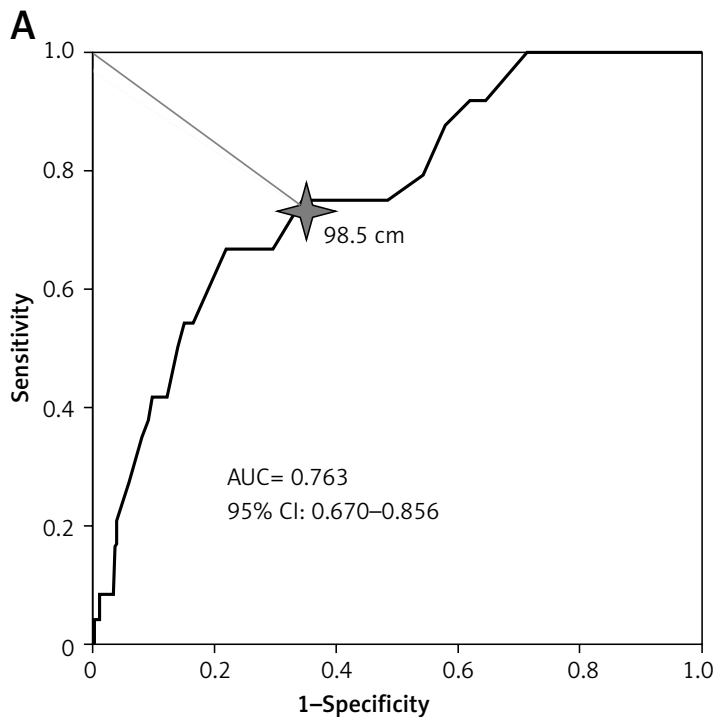

metabolic outcome for men and women, separately. The areas under the curve (AUC) showed a fair outcome for both sexes, slightly better for men (AUC $=0.763 ; 95 \% \mathrm{Cl}: 0.670-0.856$ ) than for women (AUC $=0.669 ; 95 \% \mathrm{Cl}: 0.589-0.748)$ (Figure 2). The closest data point to the upper left corner of the data plot corresponded to the cutoff points $98.5 \mathrm{~cm}$ for men and $91.5 \mathrm{~cm}$ for women, defining the optimal WC cutoffs of central obesity. These

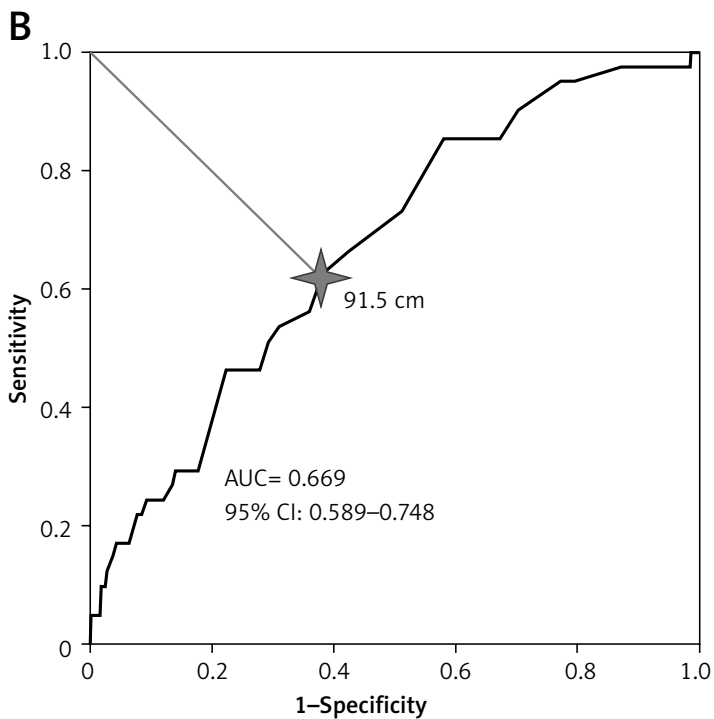

Figure 2. ROC curves testing waist circumference levels against cardiovascular outcome indexed as the detection of concomitant presence of raised FBS and hypertension during screening ( $\mathbf{A}$ - men, $\mathbf{B}$ - women)

$A \cup C$ - area under the curve; $\mathrm{Cl}$ - confidence interval.

Table II. Comparative predictive characteristics of central obesity defined by International Diabetes Federation and by new cutoff points as established by study findings, against metabolic outcome

\begin{tabular}{|c|c|c|c|c|c|c|c|c|c|c|}
\hline \multirow{2}{*}{$\begin{array}{l}\text { Classification of } \\
\text { central obesity }\end{array}$} & \multirow{2}{*}{$\begin{array}{l}\text { Sample preva- } \\
\text { lence of central } \\
\text { obesity }(\%)\end{array}$} & \multicolumn{9}{|c|}{ Predictive characteristics of central obesity against metabolic outcome } \\
\hline & & $\begin{array}{l}\mathrm{TP} \\
(n)\end{array}$ & $\begin{array}{l}\mathrm{FN} \\
(n)\end{array}$ & $\begin{array}{l}\mathrm{TN} \\
(n)\end{array}$ & $\begin{array}{l}\mathrm{FP} \\
(n)\end{array}$ & $\begin{array}{l}\text { Sensitivi- } \\
\text { ty }(\%)\end{array}$ & $\begin{array}{l}\text { Specifici- } \\
\text { ty }(\%)\end{array}$ & $J$ & $\kappa$ & $P$-value * \\
\hline \multicolumn{11}{|l|}{$\operatorname{Men}(n=536)$ : } \\
\hline $\begin{array}{l}\text { IDF recommended } \\
(\mathrm{WC} \geq 94 \mathrm{~cm})\end{array}$ & 55.20 & 19 & 5 & 235 & 277 & 79.20 & 45.90 & 0.25 & 0.039 & 0.01 \\
\hline $\begin{array}{l}\text { Study-based } \\
\text { recommendation } \\
(W C \geq 99 \mathrm{~cm})\end{array}$ & 36.20 & 18 & 6 & 336 & 176 & 75.00 & 65.60 & 0.41 & 0.093 & $<0.001$ \\
\hline \multicolumn{11}{|l|}{ Women $(n=992)$ : } \\
\hline $\begin{array}{l}\text { IDF recommended } \\
(\mathrm{WC} \geq 80 \mathrm{~cm})\end{array}$ & 79.70 & 39 & 2 & 199 & 752 & 95.10 & 20.90 & 0.16 & 0.016 & 0.01 \\
\hline $\begin{array}{l}\text { Study-based } \\
\text { recommendation } \\
(\mathrm{WC} \geq 92 \mathrm{~cm})\end{array}$ & 40.20 & 26 & 15 & 578 & 373 & 63.40 & 60.80 & 0.24 & 0.047 & $<0.001$ \\
\hline \multicolumn{11}{|c|}{ Men and women $(N=1,528)$ : } \\
\hline IDF recommended & 71.10 & 58 & 7 & 434 & 1029 & 89.20 & 29.70 & 0.19 & 0.022 & $<0.001$ \\
\hline $\begin{array}{l}\text { Study-based } \\
\text { recommendation }\end{array}$ & 38.80 & 44 & 21 & 914 & 549 & 67.70 & 62.50 & 0.3 & 0.062 & $<0.001$ \\
\hline
\end{tabular}

$T P$-true positive, FN-false negative, $T N$ - true negative, FP-false positive, J-Youden index. ${ }^{*}$-value of the $\kappa$ test between central obesity classified by IDF and newly defined cutoff points and the outcome variables. 


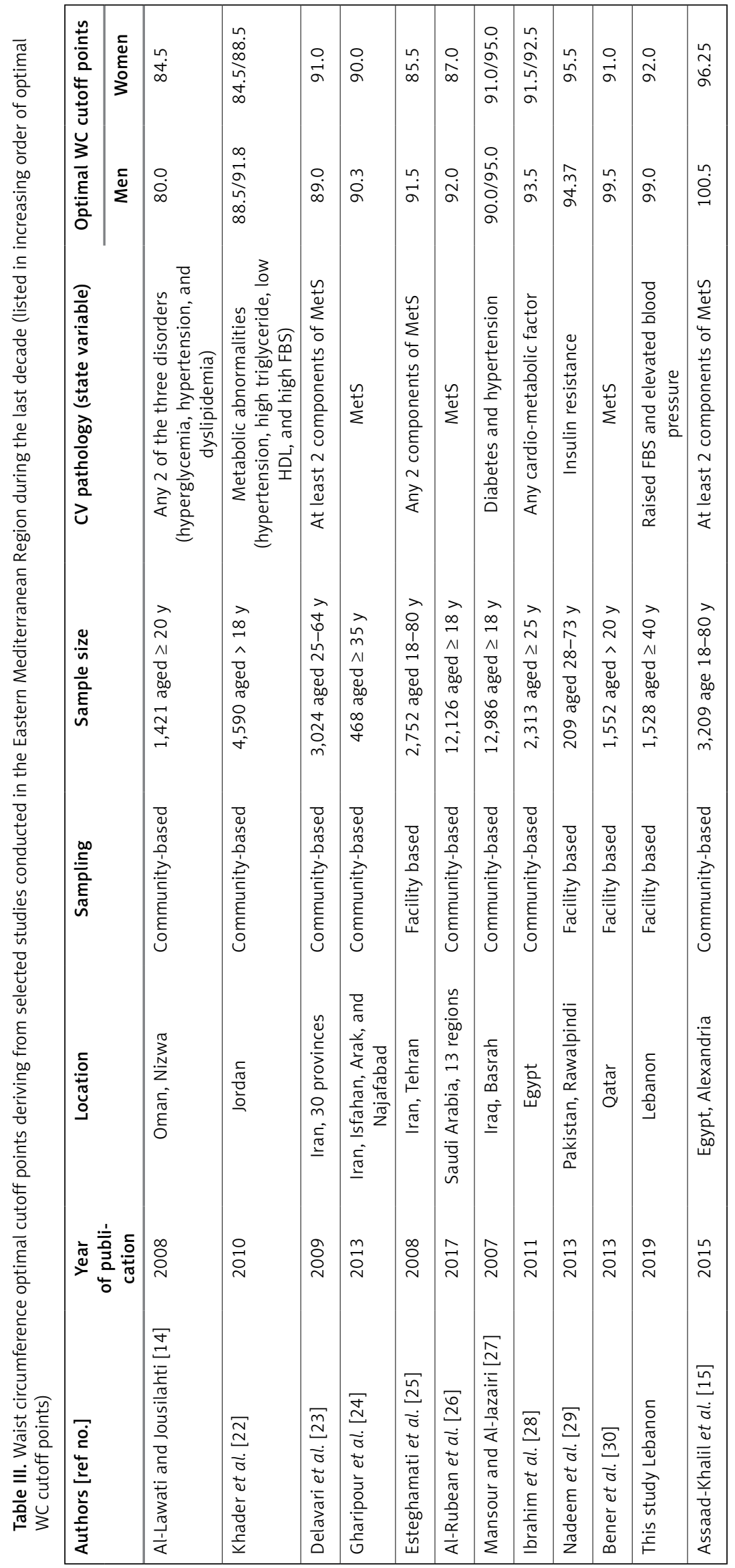


cutoff points yielded a sensitivity and a specificity of $75 \%$ and $65.6 \%$ respectively, in men, and $63.4 \%$ and $60.8 \%$ respectively, in women (Table II). When these cutoff points were used to define central obesity, the prevalence of central obesity was estimated at 38.8\% (36.2\% among men and $40.2 \%$ among women) and was lower than that estimated based on the IDF criteria $(71.1 \%$ for the total sample, 55.2\% among men and $79.7 \%$ among women). Furthermore, Youden index and $\kappa$ coefficient values were larger and superior when central obesity was defined by the study cutoff points than when they were defined according to the IDF recommendations.

\section{Discussion}

Using estimates from a large database generated as part of a screening program implemented in 25 health centers widespread in the entire Lebanese territory, this study proposes new cutoff points for WC for asymptomatic men $(98.5 \mathrm{~cm})$ and women $(91.5 \mathrm{~cm})$ aged 40 years and over. Compared to those recommended by the IDF for the region, the WC cutoffs identified through this study were higher by $4.5 \mathrm{~cm}$ and $11.5 \mathrm{~cm}$, for men and women, respectively, and had better discriminatory power to characterize individuals with metabolic outcome. Moreover, the obtained difference between sexes was narrower than that of the IDF criterion (7 cm as compared to $14 \mathrm{~cm}$, respectively). The WC cutoff points recommended in our study seemed to provide a less inflated estimate of the prevalence of central obesity than that of the IDF and may be more appropriate to use for screening among the Lebanese population for risk of CVD.

Country-specific variations in the optimal cutoff point in the WC have also been seen in studies from the region and outside, such as India [18], South Africa [19], Spain [20], and Turkey [21]. Table III and Figure 3 reproduce data generated from

A

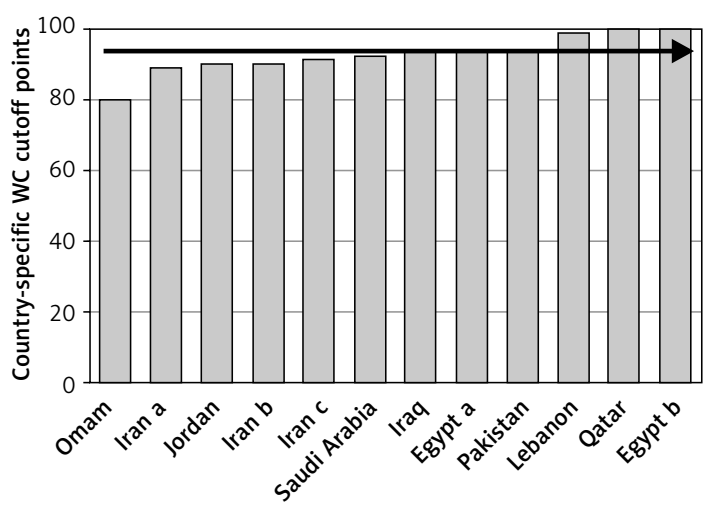

studies conducted in the Eastern Mediterranean Region during the past decade. These findings show that WC measurement is ethnically and geographically specific and cutoff points cannot be extrapolated from one context to the other. Furthermore, and in contrast to the IDF cut-off points, differentials between men and women appeared to be narrower (range: $0.3-8 \mathrm{~cm}$ vs. $14 \mathrm{~cm}$ in IDF), with several countries tolerating higher values of normal WC in women.

This study is the first to examine the WC cutoff points within the Lebanese context, yet the findings need to be considered with caution. Firstly, the study subjects included participants attending primary health care centers, and the sample was recruited based on non-probability purposive sampling; hence, the findings may not be representative of the total population. Secondly, data on lipid measurements were not available, thus limiting our choice of the metabolic outcome measure to blood pressure and FBS impairments. The lack of data on the study subjects' lipid profile poses another limitation in this study, and future studies need to examine the prognostic characteristics of central obesity and WC cut-off points in relation to the different components of lipid impairment in the Lebanese population. This is of crucial importance because dyslipidemia is among the significant risk factors for ischemic heart diseases and lipid profile testing is an expensive procedure, which limits its use in population-wide screening. Finally, whilst participants in the study were restricted to individuals aged 40 years and over, representing the age group at highest risk for diabetes and hypertension, younger age groups can present metabolic impairments as well, and would benefit from further optimization studies of WC cutoff points to define their central obesity status.

In conclusion, to date, available evidence on the prevalence of metabolic syndrome in Lebanon has been limited to the use of internationally de-

B

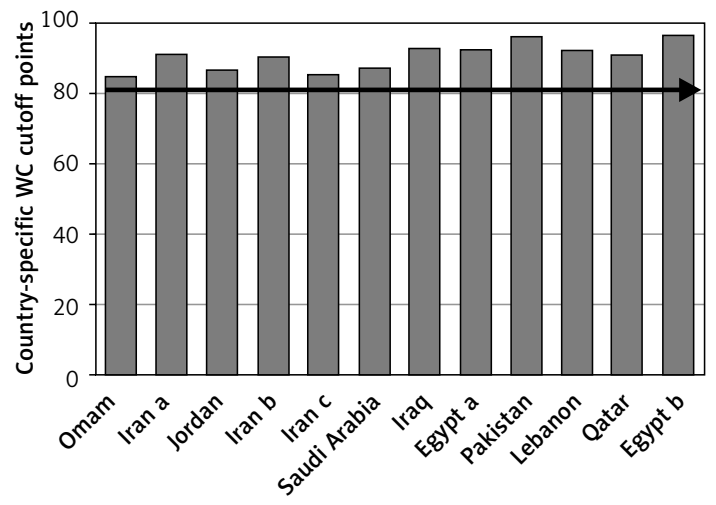

Figure 3. Optimal cutoff points as defined in countries of the region during the last decade (A - men, $\mathbf{B}-$ women). The horizontal lines represent the IDF recommended values 
fined cutoff criteria for WC (IDF or Adult Treatment Panel (ATP)). Findings from this study show that the IDF default WC cutoff points recommended by WHO for the region may be inappropriate for the Lebanese population. We identified new cutoffs for predisposition to CVD risk indexed as the concomitant presence of raised blood sugar and hypertension in otherwise healthy individuals. Further studies are needed to examine optimal WC cut-off points at the population level and on a nationally representative sample with outcomes that include lipid impairments and enhanced assessment of cardiometabolic risk factors.

\section{Conflict of interest}

The authors declare no conflict of interest.

\section{References}

1. Clark H. NCDs: a challenge to sustainable human development. Lancet 2013; 381: 510-1.

2. Rahim HFA, Sibai A, Khader Y, et al. Non-communicable diseases in the Arab world. Lancet 2014; 383: 356-67.

3. Alberti K, Eckel RH, Grundy SM, et al. Harmonizing the metabolic syndrome: a joint interim statement of the International Diabetes Federation task force on epidemiology and prevention; National Heart, Lung, and Blood Institute; American Heart Association; World Heart Federation; International Atherosclerosis Society; and International Association for the Study of Obesity. Circulation 2009; 120: 1640-5.

4. International Diabetes Federation 2006. The IDF consensus worldwide definition of the metabolic syndrome. https://www.idf.org/component/attachments/ attachments.html?id=705\&task=download. Accessed March6, 2018.

5. Beydoun MA, Kuczmarski MTF, Wang Y, Mason MA, Evans MK, Zonderman AB. Receiver-operating characteristics of adiposity for metabolic syndrome: the Healthy Aging in Neighborhoods of Diversity across the Life Span (HANDLS) study. Public Health Nutrition 2011; 14: 77-92.

6. De Koning L, Merchant AT, Pogue J, Anand SS. Waist circumference and waist-to-hip ratio as predictors of cardiovascular events: meta-regression analysis of prospective studies. Eur Heart J 2007; 28: 850-6.

7. Haghighatdoost F, Amini M, Feizi A, Iraj B. Are body mass index and waist circumference significant predictors of diabetes and prediabetes risk: results from a population based cohort study. World J Diabetes 2017; 8: 365-73.

8. Huxley R, Mendis S, Zheleznyakov E, Reddy S, Chan J. Body mass index, waist circumference and waist: hip ratio as predictors of cardiovascular risk - a review of the literature. Eur J Clin Nutr 2010; 64: 16-22.

9. Mirmiran P, Esmaillzadeh A, Azizi F. Detection of cardiovascular risk factors by anthropometric measures in Tehranian adults: receiver operating characteristic (ROC) curve analysis. Eur J Clin Nutr 2004; 58: 1110-8.

10. Schneider HJ, Glaesmer H, Klotsche J, et al. Accuracy of anthropometric indicators of obesity to predict cardiovascular risk. J Clin Endocrinol Metabol 2006; 92: 589-94.

11. Lear S, James P, Ko G, Kumanyika S. Appropriateness of waist circumference and waist-to-hip ratio cutoffs for different ethnic groups. Eur J Clin Nutr 2010; 64: 42-61.
12. Wang Z, Ma J, Si D. Optimal cut-off values and population means of waist circumference in different populations. Nutr Res Rev 2010; 23: 191-9.

13. World Health Organization 2011. Waist circumference and waist-hip ratio: report of a WHO expert consultation, Geneva, 8-11 December 2008. http://whqlibdoc. who.int/publications/2011/9789241501491_eng.pdf. Accessed January18, 2018.

14. Al-Lawati JA, Jousilahti P. Body mass index, waist circumference and waist-to-hip ratio cut-off points for categorisation of obesity among Omani Arabs. Public Health Nutr 2008; 11: 102-8.

15. Assaad-Khalil SH, Mikhail MM, Aati TA, et al. Optimal waist circumference cutoff points for the determination of abdominal obesity and detection of cardiovascular risk factors among adult Egyptian population. Indian J Endocrinol Metabol 2015; 19: 804-10.

16. Shabnam AA, Homa K, Reza MT, Bagher L, Hossein FM, Hamidreza A. Cut-off points of waist circumference and body mass index for detecting diabetes, hypercholesterolemia and hypertension according to National Non-Communicable Disease Risk Factors Surveillance in Iran. Arch Med Sci 2012; 8: 614-21.

17. Yamout R, Adib SM, Hamadeh R, Freidi A, Ammar W. Peer reviewed: screening for cardiovascular risk in asymptomatic users of the Primary Health Care Network in Lebanon, 2012-2013. Prev Chronic Dis 2014; 11: E120.

18. Midha T, Krishna V, Nath B, et al. Cut-off of body mass index and waist circumference to predict hypertension in Indian adults. World J Clin Cases 2014; 2: 272.

19. Motala AA, Esterhuizen T, Pirie FJ, Omar MA. The prevalence of metabolic syndrome and determination of the optimal waist circumference cutoff points in a rural South African community. Diabetes Care 2011; 34: 1032-7.

20. Martínez-Larrad MT, Fernández-Pérez C, Corbatón-Anchuelo A, Gabriel R, Lorenzo C, Serrano-Ríos M. Revised waist circumference cut-off points for the criteria of $a b$ dominal obesity in the Spanish population: multicenter nationwide Spanish population based study. Avan Diabetol 2011; 27: 168-74.

21. Sonmez A, Bayram F, Barcin C, Ozsan M, Kaya A, Gedik V. Waist circumference cutoff points to predict obesity, metabolic syndrome, and cardiovascular risk in Turkish adults. Int J Endocrinol 2013; 2013: 767202.

22. Khader YS, Batieha A, Jaddou H, Batieha Z, El-Khateeb M, Ajlouni K. Anthropometric cutoff values for detecting metabolic abnormalities in Jordanian adults. Diabetes Metab Syndr Obes 2010; 3: 395-402.

23. Delavari A, Forouzanfar MH, Alikhani S, Sharifian A, Kelishadi R. First nationwide study of the prevalence of the metabolic syndrome and optimal cutoff points of waist circumference in the Middle East: the national survey of risk factors for noncommunicable diseases of Iran. Diabetes Care 2009; 32: 1092-7.

24. Gharipour M, Sarrafzadegan N, Sadeghi M, et al. Predictors of metabolic syndrome in the Iranian population: waist circumference, body mass index, or waist to hip ratio? Cholesterol 2013; 2013: 198384.

25. Esteghamati A, Ashraf H, Rashidi A, Meysamie A. Waist circumference cut-off points for the diagnosis of metabolic syndrome in Iranian adults. Diabetes Res Clin Pract 2008; 82: 104-7.

26. Al-Rubean K, Youssef AM, AlFarsi Y, et al. Anthropometric cutoff values for predicting metabolic syndrome in a Saudi community: from the SAUDI-DM study. Ann Saudi Med 2017; 37: 21-30. 
27. Mansour AA, Al-Jazairi MI. Cut-off values for anthropometric variables that confer increased risk of type 2 diabetes mellitus and hypertension in Iraq. Arch Med Res 2007; 38: 253-8.

28. Ibrahim MM, Elamragy AA, Girgis H, Nour MA. Cut off values of waist circumference and associated cardiovascular risk in Egyptians. BMC Cardiovasc Disord 2011; 11: 53.

29. Nadeem A, Naveed AK, Hussain MM, Raza SI. Cut-off values of anthropometric indices to determine insulin resistance in Pakistani adults. J Pak Med Assoc 2013; 63: 1220-5.

30. Bener A, Yousafzai MT, Darwish S, Al-Hamaq AO, Nasralla EA, Abdul-Ghani M. Obesity index that better predict metabolic syndrome: body mass index, waist circumference, waist hip ratio, or waist height ratio. J Obes 2013; 2013: 269038. 